\title{
Near-field Strong Plasmonic Resonances in Bi1.5Sb0.5Te1.8Se1.2 Topological Insulator Film
}

\section{Baoshan Guo ( $\sim$ gbs@bit.edu.cn )}

Beijing Institute of Technology https://orcid.org/0000-0003-2508-7424

\section{Huan Yao}

Beijing Institute of Technology

Ningwei Zhan

Beijing Institute of Technology

\section{Lan Jiang}

Beijing Institute of Technology

\section{Research Article}

Keywords: Plasmonics, Metamaterial, Topological insulator, Mode resonance, Near field

Posted Date: November 10th, 2021

DOI: https://doi.org/10.21203/rs.3.rs-1035749/v1

License: (9) This work is licensed under a Creative Commons Attribution 4.0 International License. Read Full License

Version of Record: A version of this preprint was published at The European Physical Journal Plus on February 1st, 2022. See the published version at https://doi.org/10.1140/epjp/s13360-022-02427-x. 


\title{
Near-field strong plasmonic resonances in $\mathrm{Bi}_{1.5} \mathrm{Sb}_{0.5} \mathrm{Te}_{1.8} \mathrm{Se}_{1.2}$ topological insulator film
}

Baoshan Guo, Huan Yao, Ningwei Zhan, Lan Jiang*

Laser Micro/Nano Fabrication Laboratory, School of Mechanical Engineering, Beijing Institute of

Technology, Beijing, 100081, China;

Email: jianglan@bit.edu.cn

\begin{abstract}
:
Topological insulators are a new class of quantum materials with metallic (edge) surface states and insulating bulk states. They exhibit various novel electronic and optical properties that make them highly promising electronic, spintronic, and optoelectronic materials. Our report confirms that the topological insulator $\mathrm{Bi}_{1.5} \mathrm{Sb}_{0.5} \mathrm{Te}_{1.8} \mathrm{Se}_{1.2}$ (BSTS) is also an effective plasmonic material in the visible and near-infrared range. A BSTS film can effectively control transmission and reflection characteristics by changing the period of the hole array. This study determined that a strong resonant surface plasmonic mode at the resonance peak can confine approximately $80 \%$ of the electromagnetic field energy is demonstrated. Higher-order (second- and third-order) resonance peaks were also found, which is critical for controlling electromagnetic waves and research into new optoelectronic devices.
\end{abstract}

Keywords: Plasmonics; Metamaterial; Topological insulator; Mode resonance; Near field

\section{Introduction}

Plasmonic metallic nanostructures have been widely employed in various optical field applications, such as near-field optics and microscopy [1, 2], biological sensors [3, 4], and solar cells [5, 6]. Because of their own subwavelength characteristics, these plasmonic nanostructures can solve the problem of scale length, and they play a key role in realizing new functions and improving optical device performance. Plasmonic surface waves are a promising information carrier for ultracompact interchip interconnects [7, 8] and all-optical data processing chips [9]. However, metallic nanostructures invariably exhibit substantial parasitic losses in the visible frequency range, which 
inevitably limits the development of metamaterials, data processing circuits, and sensors for the ultraviolet, visible, and near-infrared parts [10]. This has driven the intense search for plasmonic materials beyond noble metals.

Due to low intrinsic loss, dielectric nanostructures with a high refractive index are expected to overcome the shortcomings of metallic nanostructures $[10,11]$ and have proved to be competitive candidates for high-performance optical devices $[12,13]$. In addition, dielectric nanostructures possess inherent insulating properties that do not degrade the electrical properties of optoelectronic devices and are more compatible with existing semiconductor optoelectronic device manufacturing processes [14-17].

Dielectric nanostructures, however, have a weaker light manipulation ability at the deep subwavelength scale than plasmonic metal nanostructures. Thus, to manipulate light more effectively to obtain high-performance optical devices, the objective is to combine the excellent characteristics of metal nanostructures and dielectric nanostructures in a single nanostructure [18]. A topological insulator is a type of material that behaves as an insulator internally, although its surface is conductive, meaning that electrons can only move along the surface of the material, thus giving it the characteristics of both metal and dielectric materials. Given the strong plasmonic properties of a topological insulator, they represent a new type of nanostructure that can control the surface reflection properties in the visible light range $[18,19]$. However, previous studies have not examined the transmission properties or the near-field resonant properties of topological insulator thin films.

Using a $\mathrm{Bi}_{1.5} \mathrm{Sb}_{0.5} \mathrm{Te}_{1.8} \mathrm{Se}_{1.2}$ (BSTS) film with a hole array, we identified a new mechanism of strong surface plasmonic coupling response, which is a combination of surface optical conductivity residing in a nanoscale layer of topologically protected surface states and bulk optical conductivity related to the dispersion created by the interband transitions in the medium. On nanostructured metamaterial surfaces of BSTS in particular, we observed different orders of plasmonic resonance peaks ranging from 350 to $1500 \mathrm{~nm}$ and established that all resonance modes work similarly to a two-dimensional electron gas system [20,21] that confines most of the electromagnetic energy (up to $80 \%$ ) near the surface. Combined with the topological protection characteristics of a topological insulator, this mechanism provides an ideal method for effectively controlling the behavior of electromagnetic waves and developing new optical devices. 


\section{Simulation and discussion}

For the simulation of Finite Difference Time Domain Method (FDTD), we propose a BSTS material structure consisting of a bulk insulator with a thin metal film on its surface, as shown in Fig. 1. The insulator layer thickness is $200 \mathrm{~nm}$, and that of the metal layer is $5 \mathrm{~nm}$. The hole period $(P)$ along the laser polarization direction plays a decisive role in the plasmonic excitation mode; hence, it is mainly considered as a variable in the simulation. The period of the hole array vertical to the laser polarization direction is fixed at $400 \mathrm{~nm}$, and the diameter $(D)$ of the hole is $300 \mathrm{~nm}$. The polarized laser incident is from top to bottom. The dielectric functions of the BSTS material are fitted according to [19], and the fitting curves are shown in Fig. 2.

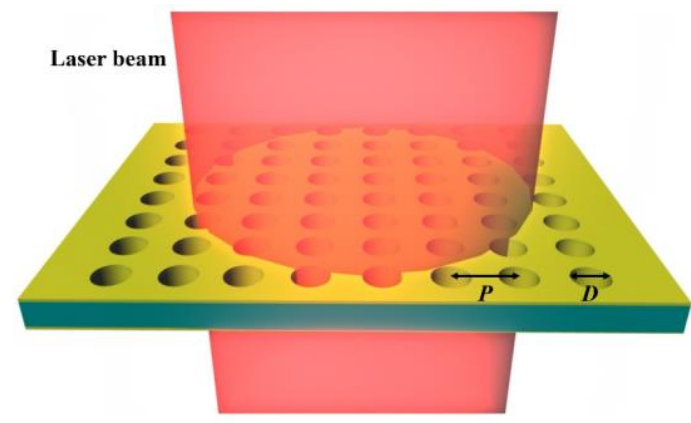

Figure 1. Schematic of BSTS film with dielectric core layer (200-nm thickness) and metallic shell layer (5-nm thickness) showing period $(P)$ and hole diameter $(D)$. The laser beam propagates top-down.

According to $\varepsilon=\varepsilon_{1}+i \varepsilon_{2}$, the complex dielectric function is divided into a real part $\varepsilon_{1}$ (blue curve in Fig. 2) and an imaginary part $\varepsilon_{2}$ (red curve in Fig. 2) that are used both to define the insulating bulk state (Fig. 2a) and the metallic surface state (Fig. 2b) of the BSTS material and to describe the interaction of incident light with the materials during the simulation.
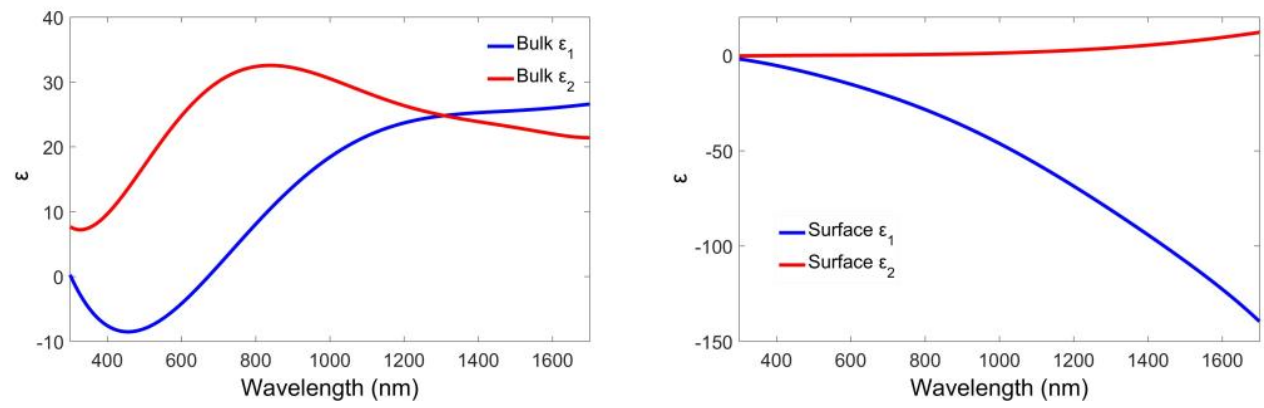

Figure 2. Optical parameters of topological insulator BSTS material. (a) Dielectric function $\varepsilon$ of the insulating bulk of BSTS film.

(b) Dielectric function $\varepsilon$ of the metallic surface of BSTS film. The blue curve represents the real part $\left(\varepsilon_{1}\right)$ and the red curve 

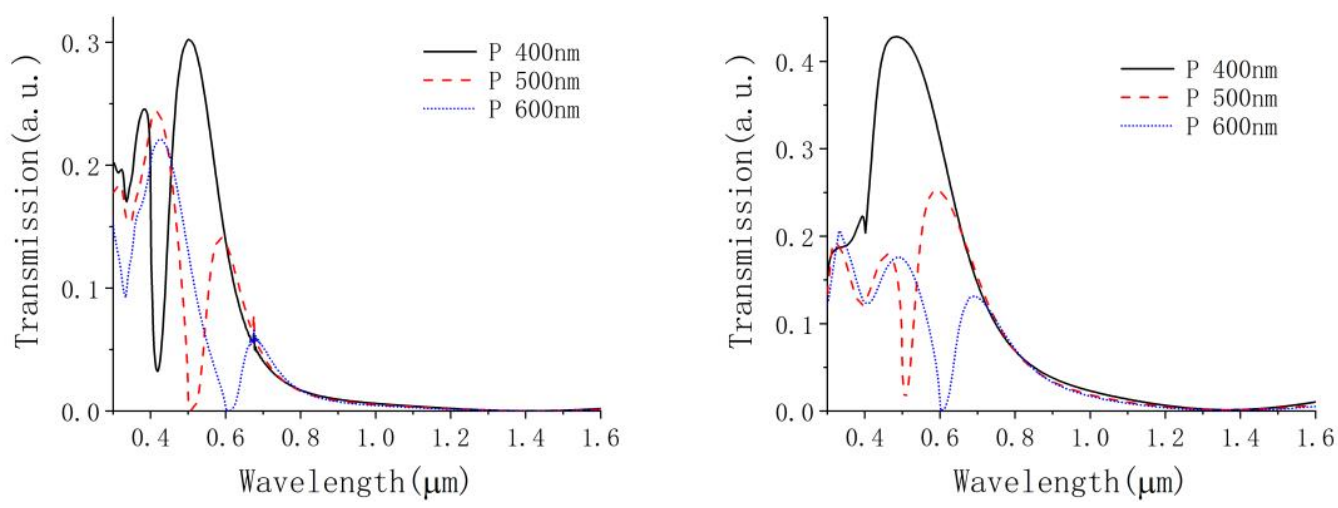

Figure 3. Transmission characteristics of BSTS film with different periods $(P)$. (a) Circle hole array: black solid curve $(P=400$ $\mathrm{nm})$; red dashed curve $(P=500 \mathrm{~nm})$; blue dotted curve $(P=600 \mathrm{~nm})$. (b) Square hole array: black solid curve $(P=400 \mathrm{~nm})$; red dashed curve $(P=500 \mathrm{~nm})$; blue dotted curve $(P=600 \mathrm{~nm})$.

The transmission characteristics of the BSTS film are shown in Fig. 3. We have changed only the period $(P)$ of the hole array along the laser polarization direction. With the increase of the period $(P)$, the shape of the transmission curve is similar but the peak position is red shifted.

From the transmission curve perspective, the position of the transmission valley is determined by the hole period $(P)$ along the laser polarization direction. For example, when $P$ is $400 \mathrm{~nm}$, the valley point is $405 \mathrm{~nm}$; when the period is $500 \mathrm{~nm}$, the valley point is $505 \mathrm{~nm}$; and when the period is 600 $\mathrm{nm}$, the valley point is $605 \mathrm{~nm}$. The position of the transmission valley corresponds to the strong resonant surface plasmonic wave-the "surface plasmonic formant." Most of the energy is thus confined at the surface, as explained in detail in the following.

With differently shaped holes, whether circular or square, the shape of the transmission curve changes (Fig. 3b), although the position of the formant point remains consistent with the period $(P)$. Hence, it can be concluded that the position of the transmission valley or surface plasmonic formant is determined by the hole period along the polarization direction. 


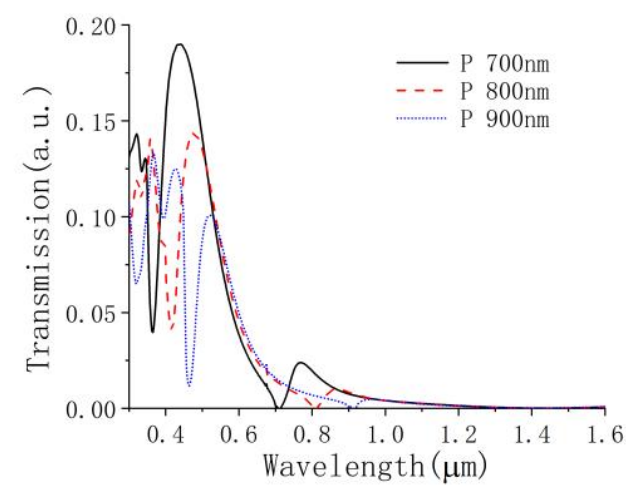

(a)

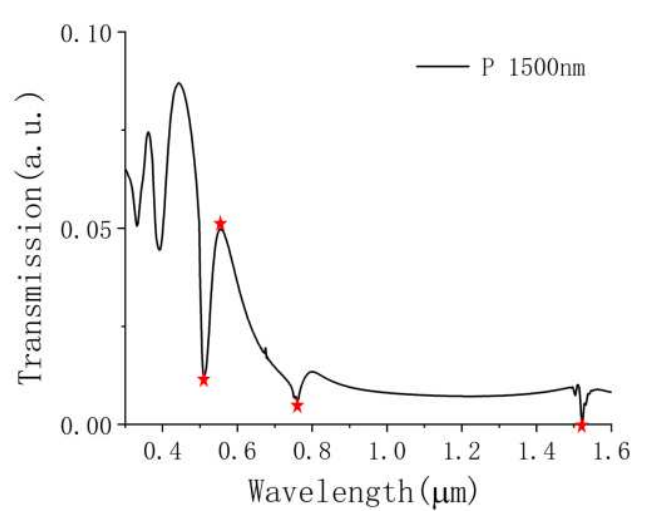

(b)

Figure 4. Transmission characteristics of BSTS film with different periods $(P)$. (a) Circle hole array: black solid curve $(P=700$ nm); red dashed curve $(P=800 \mathrm{~nm})$; blue dotted curve $(P=900 \mathrm{~nm})$. (b) Circle hole array: black solid curve $(P=1500 \mathrm{~nm})$. Three valley points and one peak point are marked by red stars.

Furthermore, when the period $(P)$ increases to more than $700 \mathrm{~nm}$ (Fig. 4a), in addition to the firstorder formant matching the period, we can clearly see the higher-order formant of frequency doubling. For example, when the first-order formant corresponding to $P=700 \mathrm{~nm}$ is at $710 \mathrm{~nm}$, the second-order formant is at $355 \mathrm{~nm}$ (black solid curve in Fig. 4a). When the first-order formant corresponding to $P=800 \mathrm{~nm}$ is at $810 \mathrm{~nm}$, the corresponding second-order formant is at $405 \mathrm{~nm}$ (red dashed curve in Fig. 4a). Also, when $P=900 \mathrm{~nm}$ ((blue dotted curve in Fig. 4a), the first-order formant is at $910 \mathrm{~nm}$ and the corresponding second-order formant is at $455 \mathrm{~nm}$.

When the period $(P)$ increases to $1500 \mathrm{~nm}$ (Fig. 4b), the first-order formant is at $1510 \mathrm{~nm}$ and the second and third resonance peaks appear at $755 \mathrm{~nm}$ and $504 \mathrm{~nm}$, respectively, corresponding to the second and third harmonic generation positions. Significant higher-order oscillation modes are thus present in the strong resonant surface plasmonic waves. 


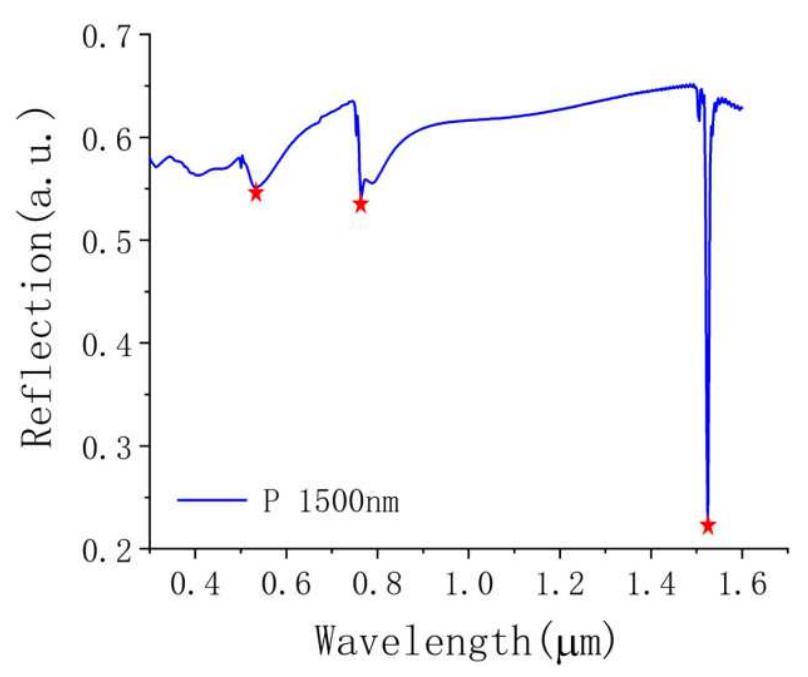

Figure 5. Reflection characteristics of BSTS film with a period of $1500 \mathrm{~nm}$. The three formants corresponding to the first,

second, and third resonance peaks are marked by red stars.

The transmission valley positions also correspond to the respective positions of the reflection valleys. The three transmission valley points of the period of $1500 \mathrm{~nm}$ (Fig. 4b) correspond to the three reflection valley points at the same positions $(504 \mathrm{~nm}, 755 \mathrm{~nm}, 1510 \mathrm{~nm}$; red stars in Fig. 5). The transmission intensity and the reflection intensity of the three formants are the lowest, demonstrating the presence of strong surface resonance modes that can effectively confine most of the electromagnetic energy to near the surface. For the formant of $1510 \mathrm{~nm}$, the transmission intensity is zero (Fig. 4b), and reflection intensity is approximately 0.2 (Fig. 5), meaning that approximately $80 \%$ of the electromagnetic energy is confined at the film surface.

To further verify the presence of resonant surface plasmonic modes, the electromagnetic field distributions $10 \mathrm{~nm}$ away from the incident and output surface of the BSTS film were investigated. Fig. 6a, 6b, 6c, and 6d illustrate the field distribution of the incident surface at the three valley points and one peak point marked by red stars in Fig. 4b. The three valley points (Fig. 6a, 6c, and 6d) all display strong resonant surface plasmonic waves, except for the peak point (Fig. 6b), which primarily exhibits radiative modes and no resonant surface modes. A similar situation occurs at the outgoing surface (OS). The OS field distributions at the same four points are shown in Fig. 6e, 6f, $6 \mathrm{~g}$, and $6 \mathrm{~h}$, respectively. Strong resonance modes also manifest at the three resonance peaks (504 $\mathrm{nm}, 755 \mathrm{~nm}, 1510 \mathrm{~nm}$ ) on the exit surface, and the intensity of the strong resonance modes achieves its highest position at $1510 \mathrm{~nm}$. This further confirms the ability of resonance modes to control electromagnetic energy. Hence, we may conclude that BSTS film can excite the strong surface 
coupling electromagnetic field and that different high-order oscillation modes control the electromagnetic wave. According to the requirements of various practical applications, a BSTS periodic structure can be designed and different oscillation modes can be selected.

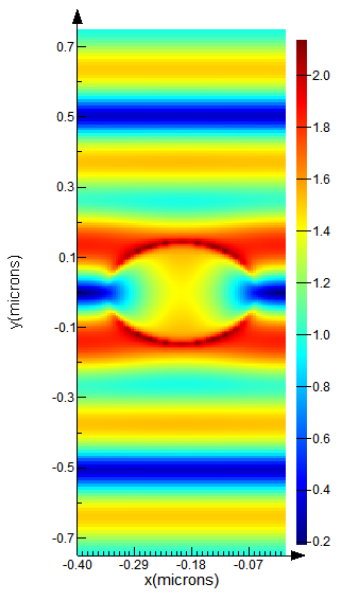

(a) IS $504 \mathrm{~nm}$,

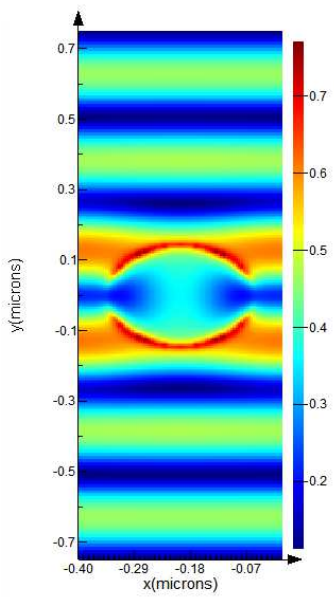

(e) OS $504 \mathrm{~nm}$,

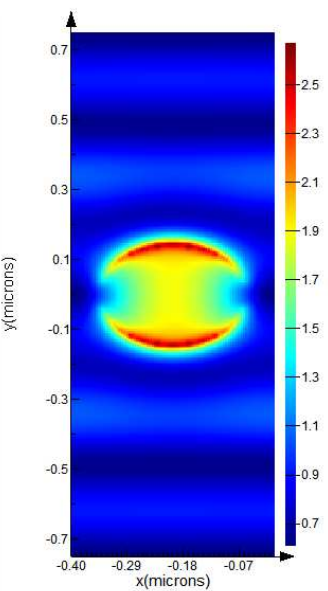

(b) IS $550 \mathrm{~nm}$,

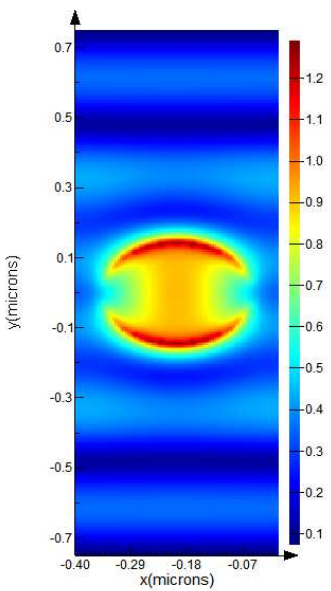

(f) OS $550 \mathrm{~nm}$,

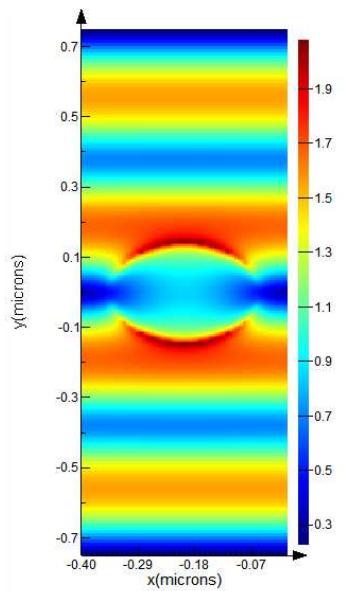

(c) IS $755 \mathrm{~nm}$,

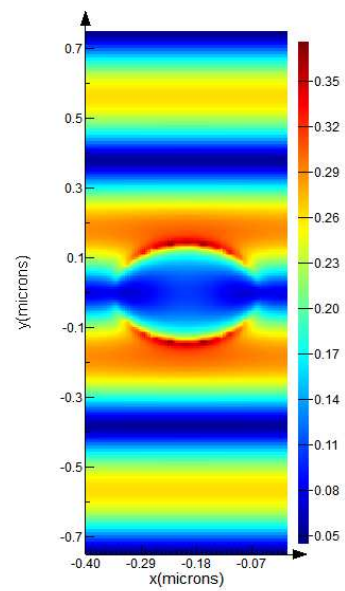

(g) OS $755 \mathrm{~nm}$,

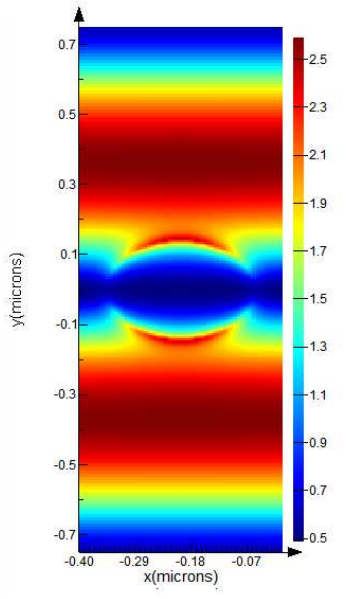

(d) IS $1510 \mathrm{~nm}$

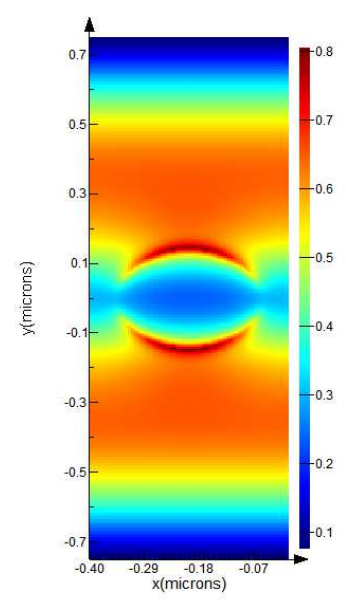

(h) OS $1510 \mathrm{~nm}$

Figure 6. Distribution of electromagnetic fields at $10 \mathrm{~nm}$ away from the incident and outgoing surfaces. (a), (b), (c), (d) are the electromagnetic fields distributions near the incident surface (IS) corresponding to the four positions marked by red stars in Fig 4b. (e), (f), (g), (h) are the electromagnetic fields distributions near the outgoing surface (OS) corresponding to the four positions marked by red stars in Fig. 4b.

\section{Conclusion}

A BSTS film with a periodic hole array was examined. It was discovered that the position of the resonance peak is consistent with the hole period along the direction of the laser polarization but is not related to the shape of the hole. Higher-order (such as second- and third-order) resonance peaks 
correspond to a half period and one-third period, depending on circumstances. Moreover, a strong coupling surface plasmonic mode occurs at the resonance peak. This strong coupling mode can confine approximately $80 \%$ of the electromagnetic field energy to the BSTS film surface. The observed plasmonic response is attributed to the combination of bulk charge carriers from interband transitions and surface charge carriers of the topological insulator. Combined with the topological protection characteristics of topological insulators, this provides an ideal method for effectively controlling the behavior of electromagnetic waves and developing new optoelectronic devices.

Funding: This study was supported by the National Natural Science Foundation of China under Grant No. 51975054 and Grant No. 61605140, and the Beijing Institute of Technology Research Fund Program for Young Scholars.

Conflicts of Interest: The authors declare no conflict of interest.

Data Availability Statement: The data presented in this study are available on request from the corresponding author.

Code Availability: Not applicable.

Authors' contributions: Conceptualization, B.G. and L.J.; methodology, B.G., H.Y. and N.Z.; investigation, B.G.; writing—original draft preparation, B.G.; writing—review and editing, B.G. and L.J.; visualization, B.G., H.Y. and N.Z.; supervision, L.J.; project administration, B.G.; funding acquisition, B.G. All authors have read and agreed to the published version of the manuscript.

Ethics approval: Not applicable.

Consent to participate: Not applicable.

Consent for publication: Not applicable.

\section{References}

1. Kawata, S.; Inouye, Y.; Verma, P. Plasmonics for near-field nano-imaging and superlensing. Nat Photon 3, 388-394 (2009).

2. Gramotnev, D. K.; Bozhevolnyi, S. I. Plasmonics beyond the diffraction limit. Nat Photon 4, 83-91 (2010).

3. Anker, J. N.; Hall, W. P.; Lyandres, O.; Shah, N. C.; Zhao, J.; Duyne, R. P. V. Biosensing with 
plasmonic nanosensors. Nat Mater 7, 442-453 (2008).

4. Liu, N.; Mesch, M.; Weiss, T.; Hentschel, M.; Giessen, H. Infrared perfect absorber and its application as plasmonic sensor. Nano Lett. 10, 2342-2348 (2010).

5. Atwater, H. A.; Polman, A. Plasmonics for improved photovoltaic devices. Nat Mater 9, 205-213 (2010).

6. Schuller, J. A.; Barnard, E. S.; Cai, W.; Jun, Y.C.; White, J. S.; Brongersma, M. L. Plasmonics for extreme light concentration and manipulation. Nat Mater 9, 193-204 (2010).

7. Ozbay, E. Plasmonics: merging photonics and electronics at nanoscale dimensions. Science 311, 189-193 (2006).

8. Miller, D. Device requirements for optical interconnects to silicon chips. Proc. IEEE 97, 11661185 (2009).

9. Almeida, V. R.; Barrios, C. A.; Panepucci, R. R.; Lipson, M. All-optical control of light on a silicon chip. Nature 431, 1081-1084 (2004).

10. Kang, G.; Yoo, J.; Ahn, J.; Kim, K. Transparent dielectric nanostructures for efficient light management in optoelectronic applications. Nano Today 10, 22-47 (2015).

11. Brongersma, M. L.; Cui, Y.; Fan, S. Light management for photovoltaics using high-index nanostructures. Nat. Mater. 13, 451-460 (2014).

12. Zhao, Q.; Zhou, J.; Zhang, F.; Lippens, D. Mie resonance-based dielectric metamaterials. Mater. Today 12, 60-69 (2009).

13. Fink, Y.; Winn, J. N.; Fan, S.; Chen, C.; Michel, J.; Joannopoulos, J. D.; Thomas, E. L. A dielectric omnidirectional reflector. Science 282, 1679-1682 (1998).

14. Cao, L.; White, J. S.; Park, J.S.; Schuller, J. A.; Clemens, B. M.; Brongersma, M. L. Engineering light absorption in semiconductor nanowire devices. Nat. Mater. 8, 643-647 (2009).

15. Lin, D.; Fan, P.; Hasman, E.; Brongersma, M. L. Dielectric gradient metasurface optical elements. Science 345, 298-302 (2014).

16. Yan, J. H.; Liu, P.; Lin, Z. Y.; Wang, H.; Chen, H. J.; Wang, C. X.; Yang, G. W. Magnetically induced forward scattering at visible wavelengths in silicon nanosphere oligomers. Nat. Commun. 6, 7042 (2015).

17. Fu, Y. H.; Kuznetsov, A. I.; Miroshnichenko, A. E.; Yu, Y. F.; Luk’yanchuk, B. Directional 
visible light scattering by silicon nanoparticles. Nat. Commun. 4, 1527 (2013).

18. Yue, Z.; Cai, B.; Wang, L.; Wang, X.; Gu, M. Intrinsically core-shell plasmonic dielectric nanostructures with ultrahigh refractive index. Sci. Adv. 2: e1501536 (2016).

19. Ou, J. Y.; So, J. K.; Adamo, G.; Sulaev, A.; Wang, L.; Zheludev, N. I. Ultraviolet and visible range plasmonics in the topological insulator $\mathrm{Bi}_{1.5} \mathrm{Sb}_{0.5} \mathrm{Te}_{1.8} \mathrm{Se}_{1.2}$. Nat. Commun. 5, 5139 (2014)

20. Sarma, S. D.; Hwang, E. Collective modes of the massless Dirac plasma. Phys. Rev. Lett. 102, $206412(2009)$.

21. Di Pietro, P.; Ortolani, M.; Limaj, O.; Di Gaspare, A.; Giliberti, V.; Giorgianni, F.; Brahlek, M.; Bansal, N.; Koirala, N.; Oh, S.; Calvani, P.; Lupi, S. Observation of Dirac plasmons in a topological insulator. Nat. Nanotechnol. 8, 556-560 (2013). 